\title{
Primary Pancreatic Lymphoma: a rare entity in pancreatic mass.
}

\author{
Noor Dev Kandel, Nirmal Lamichhane \\ Department of Surgical Oncology, BP Koirala Memorial Cancer Hospital, Bharatpur, Chitwan
}

\begin{abstract}
Primary pancreatic lymphoma is an extremely rare tumor of pancreas which clinico-radiologically mimics with pancreatic adenocarcinoma. The disease is diagnosed often after radical resection of pancreas. We report a case of 63 years old Chinese gentle man with diagnosis of Primary pancreatic lymphoma who was diagnose after surgical resection. Primary pancreatic lymphoma should also be considered as differential diagnosis in pancreatic tumors. There can be some radiological clues but accurate diagnosis depends on histopathological examination. Definitive treatment still remains controversial.
\end{abstract}

Keywords: Primary pancreatic lymphoma, Surgery, Tumor, Chemotherapy.

\section{INTRODUCTION}

Non-hodgkins lymphomas are also seen involving the pancreas as primary tumor, called as primary pancreatic lymphoma (PPL). ${ }^{1}$ Primary pancreatic lymphoma is very rare variant of neoplasm seen in less than $2 \%$ of all extra nodal Non-hodgkins lymphoma and less than $1 \%$ of all pancreatic tumors. The extra nodal involvement is commonly seen is stomach, duodenum and small intestine whereas secondary invasion of pancreas from the lymph nodes is also prevalent unlike other, primary mass of pancreas mimics with pancreatic adenocarcinoma. ${ }^{2}$ Exact diagnosis are mostly achieved after surgery by histological study of the specimen. The treatment of the primary pancreatic lymphoma with chemotherapy after surgery got better prognosis then treated with alone with chemotherapy. ${ }^{3}$ Till date less than 220 cases of Primary pancreatic lymphoma (PPL) have been reported globally out of which 39 cases are reported from People's Republic of China. The Primary pancreatic lymphoma is extremely rare entity, less than $2 \%$ of extra nodal malignant lymphomas and $0.5-0.7 \%$ of all pancreatic masses. Studies shown that there is a strong male predominance (Male to female ratio of 5:1). ${ }^{5}$ The patient mean age is $45-55$ year. $^{5}$ After excluding all possible secondary lymphomas, it is a histological diagnosis on the basis of prior clinical suspicion. ${ }^{6}$

\section{CASE PRESENTATION}

The patients 63 years old gentle man form mainland china presented outpatients clinic with history of abdominal pain for 3 weeks. Pain was insidious in onset, central upper in location, moderate to severe in intensity and constant in nature. No radiation was present. There was some weight loss but no obvious loss of appetite was present. On physical examination, built was average, no jaundice was present. On physical examination, abdomen was soft and non tender. A firm mass was felt at upper abdomen, peri umbilical region.

Ultrasonogram of the abdomen revealed a $6 \mathrm{~cm}$ mass in the head of pancreas (HOP) area. CT findings (Figure 1A) showed well defined contours of tumor with heterogenocity in texture with central necrosis in the HOP area. The contrast enhancement was significant. In MRI (Figure 1B), the mass is shown at pancreatic head with low signal intensity on T1- and intermediate signal intensity on T2- weighted images and shows faint contrast enhancement. MRCP shows dilatation of biliary tree with terminal tapering of the bile duct and dilatation of pancreatic duct (Figure 1C).

\section{Correspondence}

Nirmal Lamichhane, Department of Surgical Oncology, BP Koirala Memorial Cancer Hospital Bharatpur, Chitwan, Email: nlamichhane@hotmail.com 


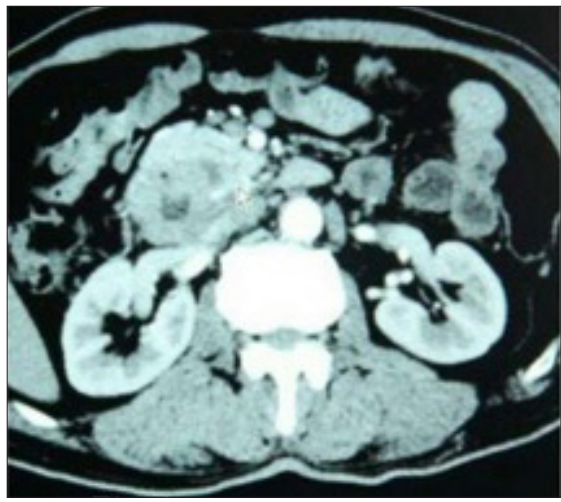

Figure 1A. CT scan of the Abdomen showing a heterogeneously enhancing mass at head of pancreas with some central necrotic area.

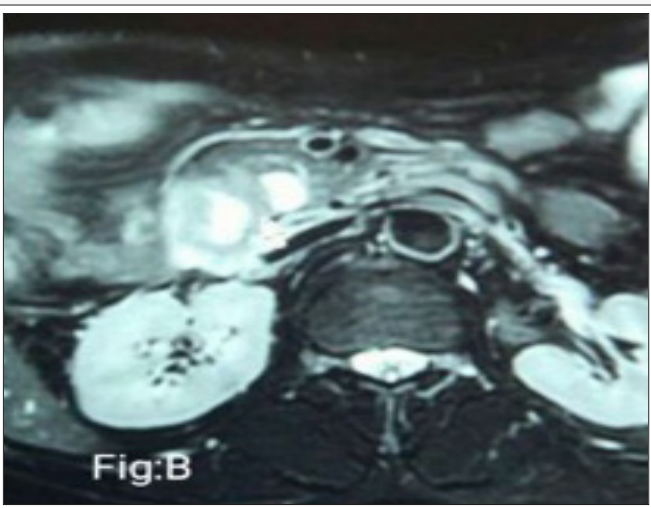

Figure 1B. MRI scan of the same the mass is shown at pancreatic head with low signal intensity on $\mathrm{T} 1$.

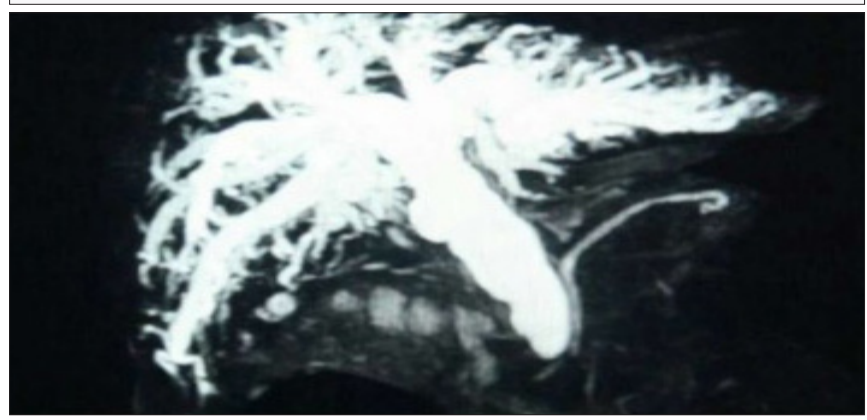

Figure 1C. MRCP shows dilatation of biliary tree with terminal tapering of the bile duct and dilatation of pancreas.

Considering the mass in the head of pancreas, patient underwent pancreaticoduodenectomy with uneventful recovery. The histopathology report there after showed B cell lymphoma. Further immunohistochemistry of the specimen showed CD 20 positivity and MUM 1 positivity which conformed the diagnosis. (Figure 2A, $2 \mathrm{~B}, 2 \mathrm{C}$ and $2 \mathrm{D}$ )

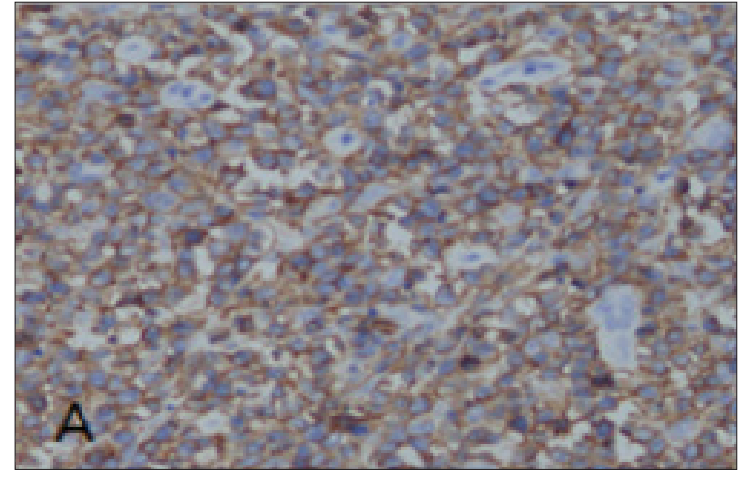

Figure 2A. Tumor cells show strong membrane staining for the $B$ cell marker protein $(C D 20, \times 40)$

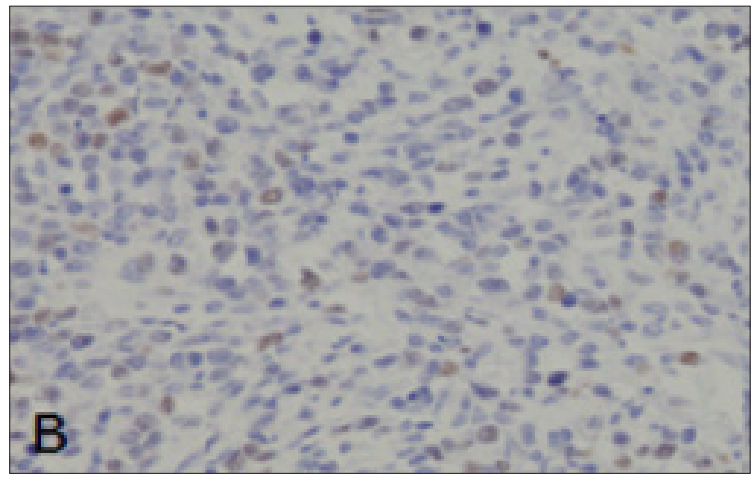

Figure 2B. Tumor cells nucleus shows brown pigments (MUM1, $\times 40$ )

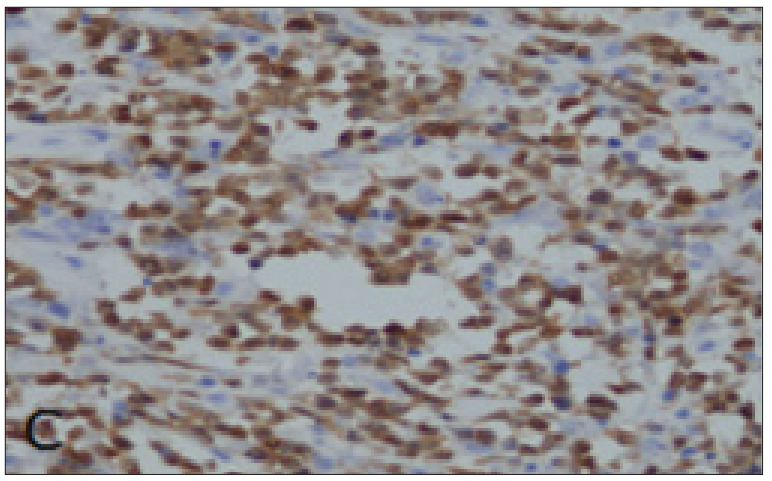

Figure 2C. Tumor cells show cytoplasm and cell membrane staining for the B cell marker protein $(P A X 5, \times 40)$

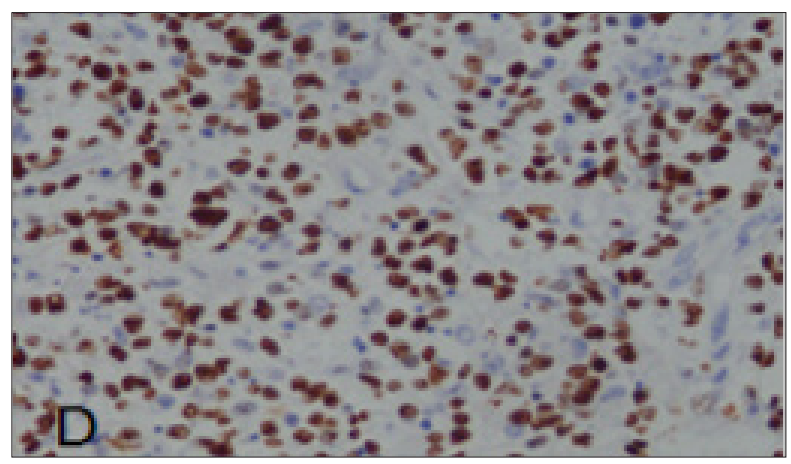

Figure 2D. Tumor cells show staining for the B cell marker protein (ki-67(+80\%), x40) 
Patient then received $\mathrm{CHOP}$ regimen chemotherapy and surviving in the 24 months after treatment.

\section{Discussion:}

The clinical features of isolated Non-hodgkin's lymphoma of pancreas present predominantly as mass in head of pancreas. The mass of primary pancreatic lymphoma is most commonly seen in head of pancreas $(>80 \%)$; and range in the size of $5-17 \mathrm{~cm}$, but size above $10 \mathrm{~cm}$ is unlikely. ${ }^{7}$ Although the clinical presentation of primary pancreatic lymphoma is nonspecific, some findings which strengthen the suspicion of lymphoma rather than the adenocarcinoma are abdominal pain, the most common presenting symptoms associated with abdominal mass. ${ }^{8}$ Abdominal pain and abdominal mass are two major symptoms which present in $83 \%$ and $58 \%$ of PPL cases, respectively. ${ }^{1}$ Two of six of our patients (33\%) presented abdominal mass others reported that only $1.3 \%$ of patients with adenocarcinoma presented as abdominal mass. ${ }^{1}$ The other symptoms are weight loss, jaundice, acute pancreatitis, acute bowel obstruction, weight loss, diarrhea, nausea, vomiting, fever, chills, night sweats and Gastrointestinal bleeding. Repeated episodes of unconsciousness have also been reported. Unconsciousness here may be related with low blood glucose level (hypoglycemic crisis) as a result of pancreatic tissue destruction by tumor infiltration. The presence of ascites is a rare finding in general and was reported only in two case reports. ${ }^{1,9}$

B-cell type only has been reported in the western countries which are the most predominant type but some cases of T-cell type has also been reported in Japanese series. Most of the cases are intermediate or high grade NHL, with diffuse large cell lymphoma being the predominant (about $60 \%$ ) histological type. ${ }^{7}$ Subtypes of low grade lymphoplasmacytic PPL has also been reported. ${ }^{10}$ The most frequent genetic alteration is translocation $t(2 ; 5)$ (p23;q35) between the anaplastic lymphoma (ALK) gene on chromosome 2 and the nucleophosmin (NPM) gene on chromosome 5 in these lymphomas. Result of this translocation, the hybrid (NPM-ALK) gene promotes the production of chimeric NPM-ALK protein. ${ }^{11}$ The NPM-ALK fusion chimeric protein can be detected immunohistochemically using monoclonal or polyclonal antibodies by RT-PCR and FISH. ${ }^{11}$

NHL is categorized on the basis of tumour burden. The
Ann Arbor staging system is the most popular system for classifying NHL. ${ }^{4}$ Behrns and associates formulated the criteria for primary pancreatic lymphoma. ${ }^{4}$ Their diagnostic criteria of PPL include: mass predominantly within the pancreas with grossly involved lymph nodes confined to the peripancreatic region, no palpable superficial lymphadenopathy, no hepatic or splenic involvement, no mediastinal nodal enlargement on chest radiograph, and normal white cell count. ${ }^{4}$ Presenting symptoms are non-specific, typically including abdominal pain, weight loss, nausea and vomiting; but also jaundice, acute pancreatitis, and small bowel obstruction. $^{1}$

Table 1 : Ann Arbor Staging fo Non hodgkins lymphoma NHL including PPL

\begin{tabular}{|c|l|}
\hline Stage & \multicolumn{1}{|c|}{ Description } \\
\hline Stage1 & $\begin{array}{l}\text { NHL is limited to one lymph node group } \\
\text { (neck, axilla, groin etc.) above or below the } \\
\text { diaphragm, or NHL is in an organ or site } \\
\text { other than the lymph nodes ( extra-nodal) } \\
\text { but has not spread to other organs of lymph } \\
\text { nodes. }\end{array}$ \\
\hline Stage 2 & $\begin{array}{l}\text { NHL is limited to two lymph node groups } \\
\text { on the same side of diaphragm, or in one } \\
\text { extra nodal organ and has spread to one or } \\
\text { more lymph node groups on the same side } \\
\text { of the diaphragm }\end{array}$ \\
\hline Stage 3 & $\begin{array}{l}\text { NHL is in two lymph node groups, with or } \\
\text { without partial involvement of of an extra } \\
\text { nodal organ or site above and below dia- } \\
\text { phragm. }\end{array}$ \\
\hline Stage 4 & $\begin{array}{l}\text { NHL is extensive( diffuse) in one organ or } \\
\text { site, with or without NHLin distant lymph } \\
\text { nodes. }\end{array}$ \\
\hline
\end{tabular}
The serum markers associated with PPL are CA19-9, $\mathrm{LDH}$ and $\beta-2$ micro-globulin. Anecdotal case reports have been reported elevated level of CA19-9 in primary pancreatic lymphoma. Essential serum markers for the diagnosis and differential diagnosis of primary pancreatic lymphoma are Lactate dehydrogenase (LDH) and $\beta-2$ micro-globulin. When the masses are associated with an elevated CA19-9 level, clinically the differential diagnosis of primary pancreatic lymphoma from pancreatic carcinoma is extremely difficult. ${ }^{12} \mathrm{Ca} 19-9$ is the most useful tumour marker in pancreatic carcinoma, but can be misleading as it may also be elevated in other 
malignancies, particularly of the upper gastrointestinal tract. $^{13}$

CT findings in lymphoma usually shows well defined contours, tumor heterogenocity, pancreatic duct dilatation and even portal venous invasion with large tumors. The presence of homogenous pancreatic mass with diameter of more than $6 \mathrm{~cm}$ or more, infiltrating the surrounding tissue accompanied by retroperitoneal or mesenteric lymphadenopathy strongly suggest Non hodgkins lymphoma but findings in itself can not distinguish lymphoma from pancreatic carcinoma. ${ }^{1} \mathrm{CT}$ findings of Non-hodgkins lymphoma were characteristic but not specific. Prayer et al, describe invasion of tumor into surroundings, with no clear anatomical margins; infiltration of retroperitoneal or upper abdominal gastrointestinal tract as a reliable sign of Non-hodgkins lymphoma. ${ }^{3}$ This was concluded and supported by Van Beers et al., reporting a case of adjacent duodenal invasion. ${ }^{19} \mathrm{~A}$ contrast enhanced scan with helical CT in arterial phase is useful in assessing the patency of major pancreatic vessels.

In MRI, two morphologic patterns of pancreatic lymphoma are recognized in MRI: a focal wellcircumscribed form and a diffuse form. The focal form occurs in the pancreatic head in $80 \%$ of cases and has a mean size of $8 \mathrm{~cm}$. It typically has uniform low attenuation at MDCT. At MRI, it has low signal intensity on T1- and intermediate signal intensity on T2- weighted images and shows faint contrast enhancement. The diffuse form is infiltrative leading to glandular enlargement of poor definition, features that can simulate the appearance of acute pancreatitis. ${ }^{7}$ Feldman et al, reported MR imaging that are similar to CT appearance; two morphological patterns of pancreatic involvement: The wellcircumscribed tumor appears with low signal in density homogenous mass in the pancreas on T1- weighted image with subtle enhancement after gadolinium enhancement. In T2-weighted images tumor shows more heterogenous character with low to intermediate signal amplitude slightly higher than that of the residual gland but much lower than the signal in density of fluid. ${ }^{14}$ Diffuse type infiltrating pancreatic involvement shows characteristics similar to low signal in density on enhanced T1 and T2weighted images, with mild to moderate enhancement after contrast. Enhancement in diffuse infiltrating type is predominately homogenous and may include small foci of little or no contrast uptake. In MR imaging and MR cholangiopancreatography bile and pancreatic ductal dilatation can be usually assessed and even the mild pancreatic ductal dilatation is detected. ${ }^{14} \mathrm{MR}$ imaging is equivalent to CT regarding information of the peripancreatic vessels and enlarged lymphnodes. More information can be obtained when MR angiography is used.

Endoscopic retrograde cholangiopancreatography (ERCP) findings were compatible with smooth stenosis of the common bile duct and main pancreatic duct without signs of infiltration. In primary pancreatic lymphoma moderate to severe dilation of wirsungs duct can be seen, which is non specific for the diagnosis. In $42 \%$ of primary pancreatic lymphoma obstructive biliary tract dilation is seen. ${ }^{14}$ Literature on ERCP appearance of wirsungs duct is described as stricture of main pancreatic duct (10\%) to no severe proximal dilation, mild duct narrowing (50\%),duct displacement (10\%) and normal duct $(30 \%) .{ }^{14}$

PET scan plays an ever-increasing role in the management of patients with lymphoma. It is useful at the initial diagnosis and work-up to help in disease staging as well as in follow up. It can also be very helpful in evaluation of response to therapy; also been proven to be superior to the conventional imaging modalities. Many studies have reported very high sensitivity and specificity of PET in the diagnosis of lymphoma and the identification of metabolically active lesions. ${ }^{14}$ Fluoro-deoxy-glucose positron emission tomography (FDG-PET/CT) plays an important role in the diagnosis, staging, and follow-up of nodal and extranodal lymphoma. ${ }^{15}$ Although the role of FDG-PET/CT in PPL has not been fully evaluated. Pancreatic lymphoma tends to be FDG avid. ${ }^{14}$ There may be FDG uptake pattern in pancreatic lymphoma-focal nodular uptake (solitary or multifocal), segmental uptake, or diffuse uptake, mirroring the CT/MR appearances.

Fine needle aspiration cytology is thought to be easy, safe and fast diagnostic technique with high rate of accuracy. ${ }^{1}$ This avoids an exploratory laparatomy. Literature states ultrasound and CT guided biopsy provide sufficient tissue for diagnosis. ${ }^{24}$ For a definitive diagnosis, CT-guided biopsy is usually necessary, and Webb et al, report that CT-guided biopsy was performed for 4 cases of PPL, and in all cases, definitive diagnosis 
was obtained. ${ }^{2}$ However, some others, reported that it was difficult to perform CT-guided biopsy safely and, therefore, this method should be performed only by trained physicians in tertiary centers. ${ }^{1}$ The use of a CT guided fine needle aspiration (FNA) has the advantage of not requiring an open surgical procedure but has the disadvantages of sampling error, difficulty in evaluating obtained sample (false positive/false negative) and the inability to subclassify the lymphoma. ${ }^{15}$ In four of six PPL patients in current study the tissue was obtained during operation and in the other two cases the tissue was obtained by EUS- FNA. However, when compared with surgery, it is difficult to obtain enough specimen by FNA to perform immuno-histochemical analysis and may lead to false-negative result. CT or US guided FNA is a safe and rapid technique to obtain a histological diagnosis, but EUS provided close by approach to get more tissues for diagnosis. ${ }^{16}$ Technical aspect wise, during CT scan it is difficult for operators to supervise the needle path. EUS-guided tissue sampling of pancreatic masses is the superior method because it is dynamic and real-time. EUS can clearly reveal the boundary of tumor and color doppler flow imaging(CDFI) can show the vein path, sequentially guide the needle aspiration and avoid the vessel damage. Complication rate of EUS-FNA was as low as $1.6 \%$, indicating it is a safe method. ${ }^{17}$ Most reported cases of PPL form PR of china were diagnosed after surgical resection at present. ${ }^{5}$ In future, with advent of EUS and its wider availability in hospitals, there will be more studies using FNA in the future.

A definitive preoperative diagnosis is extremely difficult because it can mimic pancreatic adenocarcinoma clinically and radiographically. In recent years availability of less invasive techniques gives possible accurate diagnosis. In cases of PPL, Non-operative evaluation and biopsy of pancreatic masses can avoid the need for invasive surgery. The majority of cases of PPL in literature review were diagnosed after laparotomy, which can be avoided with non-operative biopsy and new combined modality. Radiological-guided percutaneous FNA of the pancreas is a very helpful method, but requires experienced radiologists and cytopathologists to obtain a diagnosis on a small amount of tissue. ${ }^{1}$ Endoscopic ultrasound guided biopsy significantly improved the accuracy of diagnosis. ${ }^{1}$

The study suggest that FNA coupled with flow cytometry
(FC) analysis is highly accurate in the diagnosis of PPL, thereby avoiding more invasive diagnostic procedures. In a study performed at Johns Hopkins Hospital, evaluated the role of FNA and FC in the diagnosis of PPL showed six cases were classified as large B-cell lymphomas (LBCL), showing Immunoglobulin light chain restriction and CD20 expression. One case of LBCL expressed CD10, that is a marker of follicular center cell lymphoma, another case revealed CD20 expression and kappa light chain restriction. The immunophenotype along with the cytomorphologic findings of small lymphocytes with cleaved nuclei, favored a diagnosis of a low-grade follicular center cell lymphoma. FC for one case revealed a clonal of small lymphocytes with co-expression of CD5 and CD23, this phenotype conforms a diagnosis of small B-cell lymphocytic lymphoma (SLL). ${ }^{7}$

No literature found the use of FISH especially in PPL as diagnostic tools. This technique are now used routinely of the diagnosis and management of lymphoid malignancies. Most lymphomas uses FISH technique to detect chromosome anomalies with high sensitivity and specificity in paraffin-embedded tissue and may give diagnostic and prognostic genetic information. In disorders such as Hodgkin lymphoma, hairy cell leukaemia and lymphoplasmacytoid lymphoma, although many cytogenetic abnormalities have been observed, no consistent or specific abnormalities have been identified and so, at this point in our knowledge of the genetics of these disorders, cytogenetics cannot be considered a useful test for either diagnosis or prognosis. Bone marrow biopsy should be considered in all patients even with normal blood counts to complete the staging. Bone marrow biopsy and aspiration showed a reactive bone marrow and non focal lesion or atypical lymphoid aggregate in a single case report. One of the studies in nine patients, staging was performed by CT scanning and bilateral iliac crest bone marrow biopsies. The majority of patients were found to have disseminated disease. Two patients were Stage II, two Stage III, and five Stage IV. ${ }^{2}$ Treatment of primary pancreatic lymphoma is chemotherapy, radiotherapy or combination of both depending upon the size of mass in pancreas. The choice of treatment still remains controversial: Surgery versus chemothereapy versus radiation versus combination. Some literature suggest for surgical resection in early stage, but there is no study comparing surgical and nonsurgical therapy with less invasive diagnostic modalities. ${ }^{7}$ 
The role of surgery and radiotherapy in treatment remains controversial. ${ }^{16}$ Bouvet and colleagues reported the use of radiation therapy in all cases of lymphoma of the pancreas not treated with surgery; they reported four of eight patients with long term survival after standard chemotherapy and radiotherapy. ${ }^{5}$ Local failure either from residual or recurrent disease has been noted by multiple authors and accounts for more than $85 \%$ of deaths (28 of 32). Because of these frequent local failures, additional therapies directed to local control should be considered. ${ }^{19}$ Therefore, it is very important to establish a definitive diagnosis and stage of disease, to plan the modality of treatment.

Chemotherapy is the standard choice for most patient with pancreatic lymphoma. The most common regimen is CHOP. Other regimens are R-CHOP,CVP and MACOP-B. In CD20 positive tumors, $\mathrm{R}-\mathrm{CHOP}$ regimen has shown complete response and prolong the event free survival rate. One of the largest case series reported median progression free survival (PFS) of 0.7 and 6.1 year for those without surgery as initial choice of treatment, irrespective of weather the tumor was resectable or not. ${ }^{4}$ Chemotherapy with or without radiotherapy regardless of location shows $50 \%$ of complete response rate and partial response in 30\%. Over all,46\% three years disease free survival rate reported after multi-institutional studies. For management of primary pancreatic lymphoma role of radiotherapy is ill defined. Radiation upto 40 gray used and no complication except haemorrhagic postaatinic gastritis has been reported. ${ }^{1,2}$

Obstructive jaundice in primary pancreatic lymphoma occours in $42 \%$ of cases and management of such diagnosis is controversial. Endoscopic or percutaneous stent placement is performed as primary treatment. Hart and colleagues have advocated operative decompression of the bile ducts with biliary-enteric by pass. The concept was surgical decompression allows more rapid recovery of liver function before the initiation of chemotherapy. ${ }^{1}$ Some observed rapid resolution of jaundice with a short course of radiotherapy or nonhepatotoxic chemotherapy (cyclophosphamide and prednisone). Endoscopic stenting when feasible is another viable option. ${ }^{2}$

\section{PROGNOSIS}

With advancement of the treatment approach in cases of primary pancreatic lymphoma, cure rate upto $30 \%$ are reported by literatures which is greater in comparison to adenocarcinoma. No prognostic factor till date has been described in literature. However, the Milan Cancer Institute reported that tumor burden and $\mathrm{LDH}$ levels important prognostic factors in clinical outcome primary gastrointestinal non- Hodgkin's lymphoma. Behrns et al, reported that the median survival time for single chemotherapy and radiotherapy-treated PPL patients was 13 and 22 month, respectively. While the survival time could beimproved to 26 month if combined chemotherapy with radiotherapy. ${ }^{4}$ Among eight unresected cases, seven cases were treated with combined CHOP chemotherapy and radiotherapy. ${ }^{20}$ The median survival time was 67 mo (11-191 mo). Arcari et al, reported, three patients treated with chemotherapy first line therapy and two of them received also local radiotherapy; one of them was alive with complete remission for 69 months, one died of an unrelated disease at 67 months and one died of lymphoma relapse at 88 months. Two patients underwent pancreaticoduodenectomy plus adjuvant chemotherapy, one of them died of recurrent cholangitis 8 month after surgery while the other one was alive in complete remission after 160 months. ${ }^{1}$ Recurrence as late as 18 years after achieving complete remission for PPL has also been reported. ${ }^{2}$ In Japanese study, immunophenotypic differences in outcome were observed. It was found that the 1 year survival rate for B-cell lymphoma (51.9\%)was better than that of $\mathrm{T}$ cell lymphomas $(0 \%) .{ }^{20}$ The formal statistical comparison was unable to be performed, and it is impossible to obtain an unambiguous conclusion for treatment and prognosis.

\section{CONCLUSION:}

Primary pancreatic lymphoma is an uncommon tumor of pancreas that in many aspects mimics pancreatic adenocarcinoma with different in terms of clinical progression, laboratory tests, imaging examination, response to treatment and prognosis. Preoperative diagnosis is extremely difficult.Cytology and histopathological diagnosis by ultrasound or EUS or CT guided biopsy is fundamental and considered safe and accurate. Many studies and reports suggests chemotherapy and radiation as choice of treatment for long term remission. Contrarily, many researches proved the advantages of surgery as precise pathological diagnosis, decreased tumor burden and management of obstructive jaundice. Based on literature review till date, surgery with adjuvant chemotherapy shows 
better results not only in localized but even in invasive PPL. Exploratory laparotomy should be considered in patient where definitive diagnosis cannot be obtained and unavailability of highly sophisticated less invasive diagnostic modalities. The gold standard for the differential diagnosis and treatment of PPL is not established. To clear out the controversies with respect to diagnosis and treatment modality large number of cases and prospective controlled studies are required.

\section{REFERENCES:}

1. Sadot E, Yahalom J, Do RK, Teruya-Feldstein J, Allen PJ, Gönen $\mathrm{M}$ et al. Clinical features and outcome of primary pancreatic lymphoma. Ann Surg Oncol. 2015; 22(4):1176-84.

2. Webb TH, Lillemoe KD, Pitt HA, Jones RJ, Cameron JL. Pancreatic lymphoma: is surgery mandatory for diagnosis or treatment? Ann Surg. 1989; 209:25-30.

3. Zucca E, Roggero E, Bertoni F, Cavalli F. Primary extranodal non-Hodgkin's lymphomas. Part 1:Gastrointestinal, cutaneous and genitourinary lymphomas. Ann Oncol 1997; 8:727-37.

4. Behrns KE, Sarr MG, Strickler JG. Pancreatic lymphoma: is it a surgical disease? Pancreas. 1994; 9: 662-667.

5. Yu L, Chen Y, Xing L. Primary pancreatic lymphoma: two case reports and a literature review. Onco Targets Ther. 2017; 20 (10): 1687-1694.

6. Boni L, Benevento A, Dionigi G, Cabrini L, Dionigi R. Primary pancreatic lymphoma. Surg Endosc 2002; 16: 1107-1108.

7. Nayer H, Weir E G, Sheth S, et al. Primary pancreatic lymphomas. Journal of the Pancreas, 2004; 102(3): 262-273.

8. Jones WF, Sheikh MY, McClave SA. AIDS-related non-Hodgkin's lymphoma of the pancreas. Am J Gastroenterol. 1997; 92: 335-338.

9. Carbone A. Emerging pathways in the development of AIDS-related lymphomas. Lancet Oncol. 2003; 4(1): 22-9.

10. Wu XC, Andrews P, Chen VW, Groves FD.
Incidence of extranodal non-Hodgkin lymphomas among whites, blacks, and Asians/Pacific Islanders in the United States: Anatomic site and histology differences. Cancer Epidemiology 2009; 33(5):337346.

11. Parsonnet, J, Hansen S, Rodriguez L, et al. Helicobacter pylori infection and gastric lymphoma. New England Journal of Medicine 1994; 330(18):1267-1271

12. Salvatore JR, Cooper B, Shah I, Kummet T. Primary pancreatic lymphoma: a case report, literature review, and proposal for nomenclature. Med Oncol. 2000; 17: 237-247.

13. Saif MW. Primary pancreatic lymphomas. J Pancreas 2006; 7: 262-273

14. Feldman M K, Gandhi NS. Imaging Evaluation of Pancreatic Cancer .Surgical Clinics of North America. 2016; 96 (6): 1235-1256.

15. Nishimura R, Takakuwa T,Hoshida Y, Tsujimoto M, Aozasa K. Primary pancreatic lymphoma: clinicopathological analysis of 19 cases from Japan andreview of the literature. Oncology 2001; 60:3229.

16. Hamm M, Rottger P, Fiedler C. Primary nonHodgkin lymphoma of the pancreas. Chirurg 1998; 69:301-5.

17. Harris NL, Jaffe ES, Stein H, Banks PM, Chan JK,Cleary ML, et al. A revised European-American classification of lymphoid neoplasms: a proposal from the International Lymphoma Study Group. Blood 1994;84:1361-92.

18. Prayer L, Schurawitzki H, Mallek R, Mostbeck G.CT in pancreatic involvement of non-Hodgkin lymphoma. Acta Radiol 1992; 33:123-7.

19. Van Beers B, Lalonde L, Soyer P, Grandin C, Trigaux JP, De Ronde T, et al. Dynamic CT in pancreatic lymphoma. J Comput Assist Tomogr 1993;17:94-7.

20. Ishigami K, Tajima T, Nishie A, et al. (2010) MRI findings of pancreatic lymphoma and autoimmune pancreatitis: a comparative study. Eur J Radiol. 2009; 74(3):e22-e28 